\title{
SOLAR X-RAY SOURCE UNASSOCIATED \\ WITH SUNSPOTS
}

\author{
G. A. GURZADYAN and K. V. VARTANIAN
}

Space Research Branch of Byurakan Astrophysical Observatory, Erevan, Armenian S.S.R., U.S.S.R.

On October 3, 1970 the astrophysical rocket observatory K-2 was launched from the Soviet Union, designed to investigate the Sun in the region of short- and X-ray waves Gurzadyan et al. (1971). During the launching a series of X-ray pictures of the Sun was received, in the period $04 \mathrm{~h} 22 \mathrm{~min}$ to $04 \mathrm{~h} 30 \mathrm{~min}$ universal time, through an aluminum foil $5000 \AA$ thick, covering a polistriol film $1000 \AA$ thick. The pictures were taken by means of a pinhole camera under the following conditions: the focal length was $150 \mathrm{~mm}$, the angular resolution - from $8^{\prime}$ to 0.92 , which corresponds to the dimensions of the input diaphragm from $0.28 \mathrm{~mm}$ to $0.04 \mathrm{~mm}$, effective exposure $-520 \mathrm{~s}$, the pointing accuracy by a stabilised platform carrying the instruments - $0.7-0.8$. The picture was taken by a film of the UF-R type (Kalinkina et al., 1965).

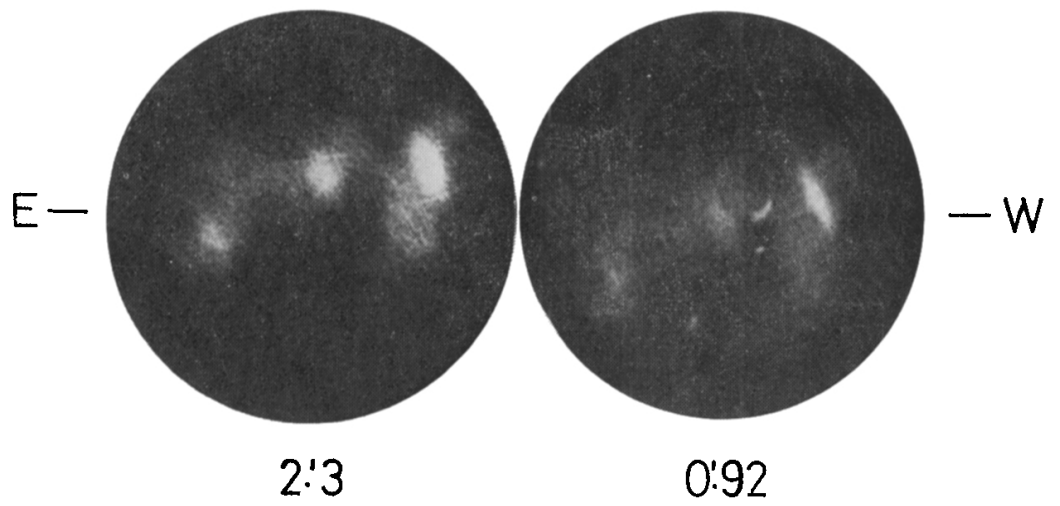

Fig, 1. X-ray pictures of the Sun (Oct. 3, 1970) in the interval of wavelengths $44-60 \AA$ with an angular resolution of $2: 3$ and 0.92 .

Figure 1 reproduces two of the thirty-eight X-ray pictures of the Sun we have obtained, while Figures 2 and 3 present their isophotes. The numbers on the isophotes designate the intensities of $\mathrm{X}$-ray radiation in arbitrary units. The angular resolution of those pictures is 2.3 and 0.92 . The characteristic curve essential for calibration of the photographic densities of the X-ray solar pictures in terms of relative intensity and, in particular, for the construction of X-ray isophotes, has been plotted with the help of the same X-ray pictures of the Sun in the following manner. A comparison has been made between the photographic densities of the same local X-ray source on the Sun for different sizes of the diaphragms, the picture of the source being obtained through 
different diaphragms. Such a method of plotting the characteristic curve for the measurement of X-ray solar pictures should be considered the most reliable and free from systematic errors of any kind.

The conspicuous fact in the above isophotes is the occurrence of three local sources of X-ray radiation - A, B and C during our observations (the shaded area in Figure 2). The emission measures of the sources differ greatly; $A$ is the most powerful source, five to six times intense than $\mathrm{C}$ and about three times more powerful than source $\mathrm{B}$ in $X$ rays.

A comparison has been made between the above X-ray isophotes and the general conditions of the Sun during our observations, which enabled us to arrive at a number of interesting conclusions.

On the day when we observed the Sun, three groups of sunspots - Nos. 432, 438, and 441 - were recorded; the total area of each group being of the order of, or greater than, $100 \times 10^{-6}$ (in units of the Sun's disk). Number 432 is the most powerful, with its total area reaching $510 \times 10^{-6}$.

The positions of the said three groups are drawn in Figures 2 and 3 (circles with crosses). It should be noted that the positions of two of these groups of spots, i.e. Nos. 438 and 441, concur fairly well with the positions of the local X-ray sources B and $\mathrm{C}$.

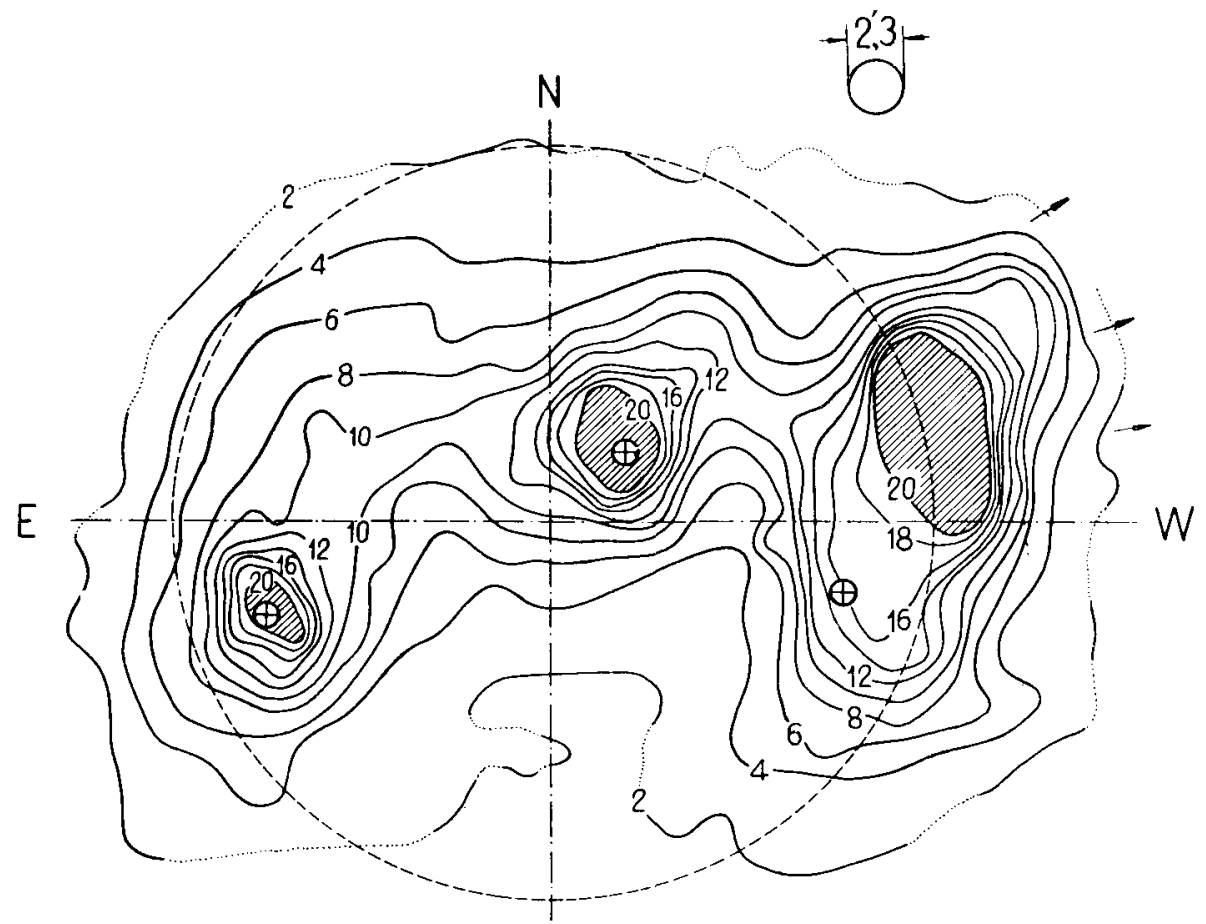

Fig. 2. X-ray isophotes of the Sun. Angular resolution 2!3. The figures on the isophotes stand for the intensity in arbitrary units; the circles with crosses mark the location of sunspot groups, while the arrows from the west indicate the position of the centre and ends of the prominence. 


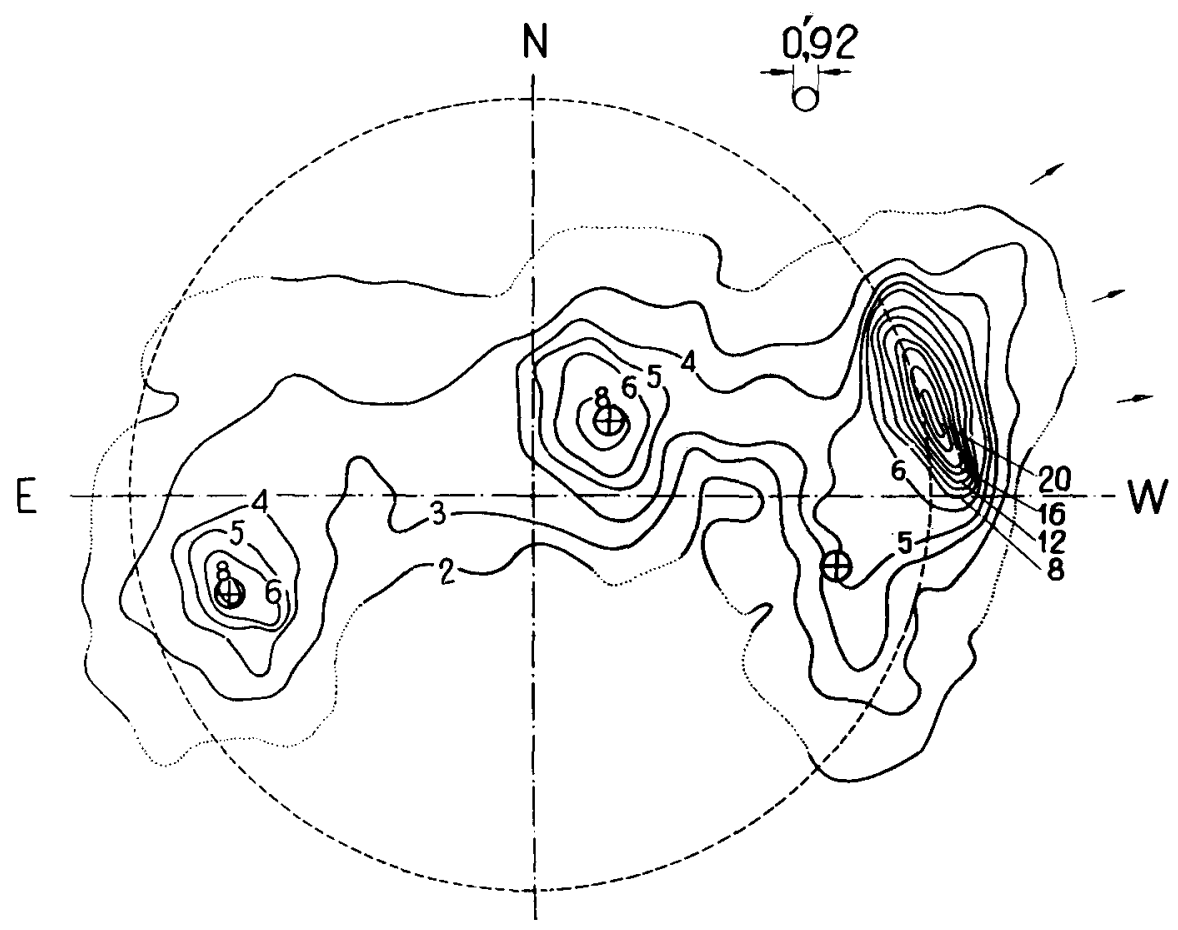

Fig. 3. X-ray isophotes of the Sun. Angular resolution 0.92 (for the remaining designations see Figure 2).

However, the following two circumstances, derived from our observations, seem to be the most significant:

(a) The largest group of sunspots, No. 432, is not associated at all with a more or less noticable region of $\mathrm{X}$-ray radiation.

(b) The most powerful X-ray source A is not associated with the sunspots in any way. Moreover, it follows from Figure 3 that the maximum radiation of this source even lies beyond the Sun's limb.

As a better illustration, plotted on the Sun's disk of Figure 4 are the X-ray sources $\mathrm{A}, \mathrm{B}$ and $\mathrm{C}$ (shaded areas with dotted boundaries). The dimensions of the drawings correspond to the power of the $\mathrm{X}$ radiation of the given source - in the first case, and to the size of the total area of sunspots - in the second case. A similar comparison of a quantitative nature, is also made in Table $\mathrm{I}$. It shows the local sources in $\mathrm{X}$ rays (column 1), the powers of these sources in arbitrary units (column 2), established by means of isophotes in Figure 3 and corresponding to levels above 5 units of the intensity; it also indicates the number of the group of sunspots (column 3 ) and the total area of sunspots (column 4). The dashes in Table I indicate that sunspots are lacking altogether within the area of the X-ray source A, while in the locality of No. 432 group of sunspots we have only a general X-ray background, with no sign whatever of the presence of a local X-ray source. 


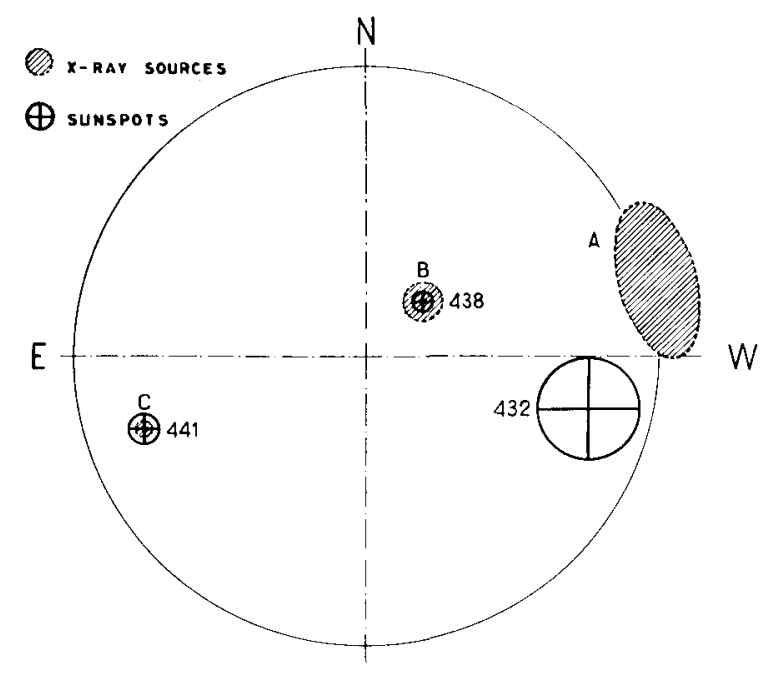

Fig. 4. Schematic recognition of X-ray sources A, B, C (the shaded areas, skirted by dotted lines) and the groups of sunspots (circles with crosses) as displayed by the Sun on October 3, 1970 in $04 \mathrm{~h}$ 26 min UT. The dimensions of X-ray sources correspond to their power, while the sizes of the group spots are in line with their total area. The figures indicate the numbers of the groups of spots according to Soln. Dann. (1970).

\section{TABLE I}

Comparison of X-ray sources $\mathrm{A}, \mathrm{B}$ and $\mathrm{C}$ with three groups of sunspots Nos. 438, 441 and 432 (October 3, 1970, 04 h, 26 min UT)

\begin{tabular}{llll}
\hline $\begin{array}{l}\text { X-ray } \\
\text { sources }\end{array}$ & $\begin{array}{l}\text { Radiation } \\
\text { intensity } \\
\text { in X-rays }\end{array}$ & $\begin{array}{l}\text { Nos. of } \\
\text { sunspot } \\
\text { groups }\end{array}$ & $\begin{array}{l}\text { Total area of } \\
\text { spots in the } \\
\text { group }\end{array}$ \\
1 & 2 & 3 & 4 \\
\hline A & 270 & - & - \\
B & 100 & 438 & $98 \times 10^{-6}$ \\
C & 46 & 441 & $142 \times 10^{-6}$ \\
- & - & 432 & $510 \times 10^{-6}$ \\
\hline
\end{tabular}

It is noteworthy that the power of X-ray radiation per unit area of the sunspots is nearly three times as large in the case of group No. 438 (source B) as in the case No. 441 (source C).

On the same day as our observations the appearance of a prominence at the western end of the Sun's disk at coordinates $\varphi=+18^{\circ}$ and $L=+90^{\circ}$ was registered at Abastumani Observatory. This corresponds to the position of the most powerful X-ray source A. Furthermore, the prominence made its appearance at $04 \mathrm{~h} 02 \mathrm{~min}$ and ended at $04 \mathrm{~h} 46 \mathrm{~min}$, the maximum of its development corresponding to the middle our observation time (04 h $26 \mathrm{~min})$. The boundaries and axis of this prominence are designated by arrows in Figures 2 and 3 (from the west side of the Sun's disk). 
Thus there are obvious signs indicating that the most powerful X-ray source is associated with the prominence. Curiously enough, the prominence does not seem to be particularly powerful; its intensity is of Type 1 (height $0.05-0.10 R_{\odot}$ ), the direction of the movement is radial. The appearance of the prominence was not associated with the appearance of solar flares.

Weak traces of the above prominence are detectable in the picture of the Sun's disk, also taken at Abastumani Observatory in the line $3934 \AA$ of ionized calcium (Figure 5). Incidentally, a comparison of this picture with the $\mathrm{X}$-ray isophotes on the one hand and the positions and total area of sunspots on the other, leads to interesting conclu-

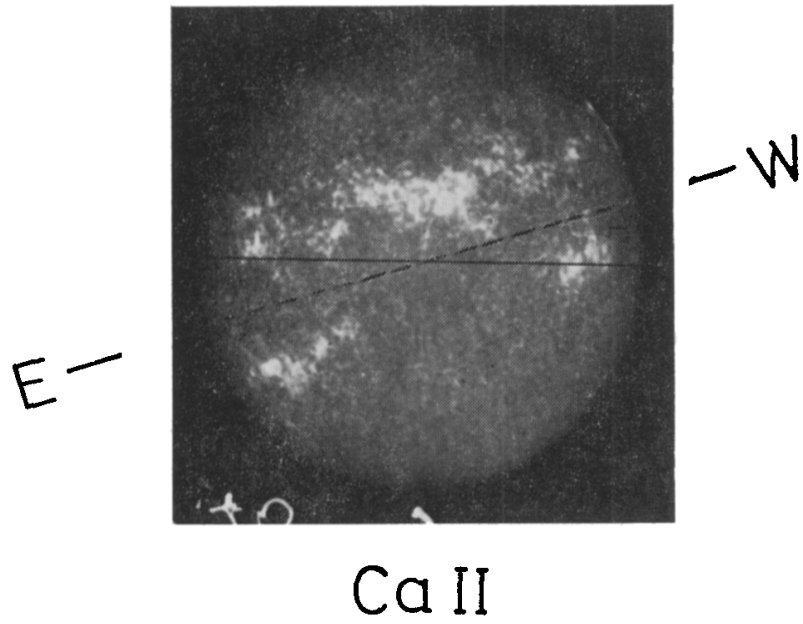

Fig. 5. Picture of the Sun taken on October 3,1970 , in the line $3934 \AA$ of ionized calcium.

sions. First of all, three regions are marked quite distinctly as active in the lines of ionized calcium, the positions of which coincide with the positions of the three groups of sunspots. However, the smallest group of spots (No. 438) corresponds to the biggest calcium region (point III), located almost in the centre of the Sun's disk; the region of calcium smallest in size (point II) agrees with the largest group of spots (No. 438). As to the X-ray sources, the positions of two of them, B and C, correspond to the two calcium regions, whereas no ionized calcium is to be found in the area of source $\mathrm{A}$.

Regarding chromospheric flares, we re-observed several cases during the day (October 3,1970 ) but none of them corresponded in time with the moment of our X-ray picture. These flares were on the whole associated with the spots of groups Nos. 438 and 441 . No chromospheric flares were detected from No. 432 all through the day.

Summing up the foregoing we come to the conclusion that at the time of our observations the most powerful X-ray source (A) on the Sun can be associated with the appearance of the prominence. On the other hand, the largest of the sunspot groups (No.432) did not prove a source of X-ray radiation of the expected power.

Furthermore, according to the preliminary estimates, the intensity of source $A$ is considerably higher than the total power of radiation in $\mathrm{X}$ rays from the entire corona. 
Even exercising a certain caution, one could state at least that the $\mathrm{X}$-ray radiation of source $\mathrm{A}$ is of a markedly non-thermal nature, otherwise a kinetic temperature should be ascribed to the plasma of the prominence, measurably exceeding the temperature of the corona. Since the temperature in the prominence is appreciably lower, the possibility of the appearance or discharge of particles (of electrons) with very high energy can be considered during the formation of the prominence. Such high energy particles (fast electrons) will radiate in the $\mathrm{X}$-ray region of the spectrum, while failing to make a significant contribution to the ordinary optical region. On the other hand, since within the prominence high magnetic fields as a rule are not available, such as are characteristic of sunspots, one cannot speak of the formation of fast electrons following from their acceleration in magnetic fields. Rather they originate from matter ejected when the prominence emerges.

The generation of powerful X-ray radiation in the region of the Sun's atmosphere, with no powerful magnetic fields (source A) on the one hand, and the lack of X-ray radiation from a region with rather powerful magnetic fields (group No. 432) on the other, gives food for pondering over the problem of whether the hypothesis of magnetic annihilation as the energy source of flares on the Sun holds or not. For years attempts have been made to interpret the generation of chromospheric, X-ray and other flares on the Sun exclusively within the framework of magnetic phenomena in terms of this hypothesis and the theories advanced with this as its basis. In the light of the foregoing considerations, the hypothesis of magnetic annihilation should, in all probability, be called into question.

\section{Acknowledgements}

In conclusion our heartfelt thanks are due to E. A. Kazarian, M. N. Krmoyan, and R. A. Epremian for preparing the equipment and conducting the experiment, to $G$. Khachtourian for preparing the X-ray filter, and to Prof. E. K. Kharadze for kindly placing the solar data at our disposal before their publication.

\section{References}

Gurzadyan, G. A., Kazarian, E. A., Krmoyan, M. N., and Epremian, R. A.: 1971, Doklady Akad. Nauk Arm. S.S.R. 53, 224.

Kalinkina, T. A., Oshurkova, A. N., Pankova, A. A., Uvarova, V. M., Tshistova, G. I., and Shpolskij, M. R.: 1965, J. Priclad. Spectrosc. 2, 475.

Soln. Dann. 1970, Bull. No. 10, October, Leningrad.

\section{DISCUSSION}

C. de Jager: About $10 \%$ of all flares observed in the visual region (in $\mathrm{H} \alpha$ ) are not associated with sunspot groups. If your bright spot is a flare the non-association with spots is not surprising. If it is a longer-lived feature we should remember that active regions without spots can often produce brilliant facular regions, which are associated with fairly dense coronal regions, which emit a strong X-ray flux. 
G. A. Gurzadyan: At the time of our observations the brilliant facular regions are absent completeJy, on the Sun's disk. At the position of the strong X-ray source A we have neither an increase nor a decrease in the intensity in the line of ionized calcium. In our case the source $A$ is connected with the prominence only. I know nothing of the generation of X rays in prominences, but I think that it will be very difficult to make a test for the explanation of such a powerful X-ray source in the framework of the thermal nature of its origin.

W. M. Glencross: University College London and Leicester University have an X-ray spectroheliograph on the satellite OSO 5 (wavelength range 3-12 $\AA$ ). We should be able to determine whether the bright emitting region had a long lifetime or whether it was a flare.

G. A. Gurzadyan: The strong X-ray source A lies on the edge of the solar limb and perhaps behind the limb. It is unlikely that this source can be identified with typical flare phenomena. The source $A$ is connected physically with the prominence and we can only suppose that the duration of this X-ray source may be the same as the duration of prominence itself, i.e. nearly $40 \mathrm{~min}$.

V. Kotov: Chromospheric flares, radio bursts and other active processes occur often in the 'quiet' photospheric regions just before the appearance of sunspots. Can you say where the sunspot group was observed in this region during the next passage of it across the disk? Have you some information about the magnetic field in this region?

G. A. Gurzadyan: About the spot groups during the next passage across the disk, I do not have any information. In the structure of the magnetic fields at the moment of our observations we have no unusual situation. 